\title{
DETERMINING ROTATIONAL DEFORMITY IN BROKEN FOREARMS
}

\author{
Y. M. STOKES ${ }^{1}$
}

(Received 14 February, 2001)

\begin{abstract}
To assess rotational deformity in a broken forearm, an orthopaedic surgeon needs to determine the amount of rotation of the radius from one or more two-dimensional $x$-rays of the fracture. This requires only simple first-year university mathematics - rotational transformations of ellipses plus a little differential calculus - which yields a general formula giving the rotation angle from information obtained from an $x$-ray. Preliminary comparisons with experimental results are excellent. This is a practical problem that may be useful to motivate the teaching of conic sections.
\end{abstract}

\section{Problem description}

The human forearm contains two bones, the ulna and the radius. The radius rotates about the ulna and governs rotational movement of the forearm [2]. Starting with the hand in a vertical position, thumb pointing up, the normal rotational range is $80^{\circ}$ outwards in supination (palm up) and $80^{\circ}$ inwards in pronation (palm down) giving a total of $160^{\circ}$ [1]. Most fractures of the radius occur in the distal radius, that is, the lower part, above the wrist. The two segments may become misaligned due to rotation and/or translation; here we focus on rotational deformity which effectively reduces the range of rotational movement of the forearm and hand in one direction. A loss of up to $30^{\circ}$ in supination or pronation can be tolerated while maintaining sufficient functionality to carry out normal activities [1] but the acceptable deformation may be less for specific individuals.

Thus an orthopaedic surgeon must assess the degree and effect of rotational deformity in a radial fracture so as to determine whether corrective surgery is warranted. This must be determined from one or more two-dimensional images of the break

\footnotetext{
'Department of Applied Mathematics, University of Adelaide, Adelaide SA 5005, Australia; e-mail: ystokes@maths.adelaide.edu.au.
}

(C) Australian Mathematical Society 2003, Serial-fee code 1446-1811/03 
obtained by $\mathrm{x}$-ray. Note that the amount of translational deformity is just the misalignment of the axes of the two segments and is directly measurable on $\mathrm{x}$-ray images. The rotational deformity is more difficult to determine; it must be computed from the measured difference in width of the two bone segments at the break position (see Figures 1 and 2), with the direction of rotation (supination or pronation) determined from the external appearance of the hand and arm. Sectioning of the distal radius shows it to be roughly elliptical in cross-section so that, by considering rotational transformations of ellipses, we may obtain an approximate formula relating the ratio of the widths of the two segments of a fractured radius as seen on an $x$-ray of the fracture, to the angle of rotation.

A request by an orthopaedic surgeon [1] for such a formula prompted this work. It requires no more than first-year university mathematics and is an interesting and easily comprehensible practical problem that might be useful to motivate the teaching of conic sections and their transformations (as, for example, in Mathematics I Algebra at the University of Adelaide).

\section{Solution}

For practical purposes we may assume that the angle of rotation will be between $-\pi / 2$ and $\pi / 2$. Then, for an ellipse with known major axis $\ell=2 a$ and minor axis $w=2 b$, we must find the angle of rotation $\theta$ given the ratio $r=W / w($ or $\rho=L / \ell$ ) determined from an $x$-ray of a break (see Figures 1 and 2).

Let the coordinate system be as shown in Figure 3 (elliptical 'radius' shown in normal unrotated position). The equation of the ellipse is

$$
\frac{x^{2}}{a^{2}}+\frac{y^{2}}{b^{2}}=\left[\begin{array}{ll}
x & y
\end{array}\right]\left[\begin{array}{cc}
1 / a^{2} & 0 \\
0 & 1 / b^{2}
\end{array}\right]\left[\begin{array}{l}
x \\
y
\end{array}\right]=1
$$

On rotating anticlockwise about the origin through angle $\theta$ the ellipse is given by

or

$$
\left[\begin{array}{ll}
x & y
\end{array}\right]\left[\begin{array}{cc}
\cos \theta & -\sin \theta \\
\sin \theta & \cos \theta
\end{array}\right]\left[\begin{array}{cc}
1 / a^{2} & 0 \\
0 & 1 / b^{2}
\end{array}\right]\left[\begin{array}{cc}
\cos \theta & \sin \theta \\
-\sin \theta & \cos \theta
\end{array}\right]\left[\begin{array}{l}
x \\
y
\end{array}\right]=1
$$

$$
\left[\begin{array}{ll}
x & y
\end{array}\right]\left[\begin{array}{ll}
A & B \\
B & C
\end{array}\right]\left[\begin{array}{l}
x \\
y
\end{array}\right]=\mathbb{R}_{r}
$$

that is,

$$
A x^{2}+2 B x y+C y^{2}=1
$$

where

$$
A=\frac{\cos ^{2} \theta}{a^{2}}+\frac{\sin ^{2} \theta}{b^{2}}, \quad B=\left(\frac{1}{a^{2}}-\frac{1}{b^{2}}\right) \cos \theta \sin \theta \quad \text { and } \quad C=\frac{\cos ^{2} \theta}{b^{2}}+\frac{\sin ^{2} \theta}{a^{2}} \text {. }
$$




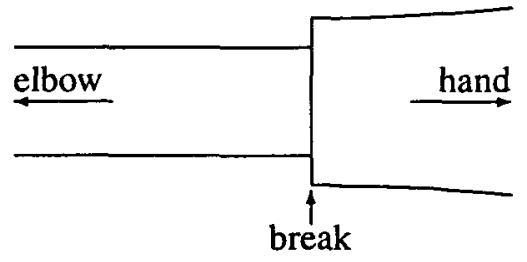

FIGURE 1. Diagrammatic $x$-ray image of break.

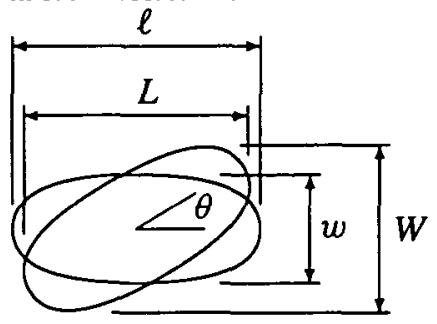

FIGURE 2. Cross-section at break; distal segment rotated through angle $\theta$.

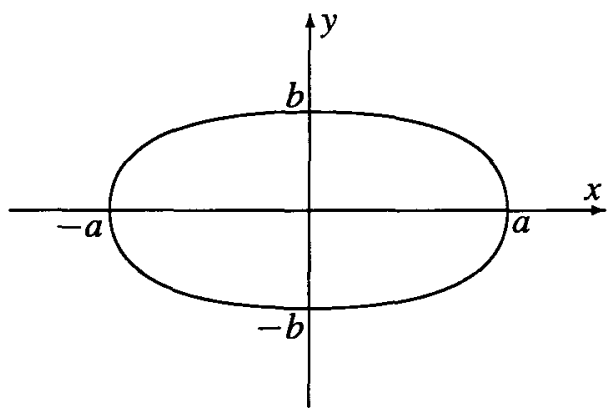

FIGURE 3. Coordinate system.

To find the width $W$ of the rotated ellipse we simply find the $y$-coordinates of the points at which $d y / d x=0$. Differentiating (2.1) with respect to $x$ gives

$$
A x+B y+\frac{d y}{d x}(B x+C y)=0,
$$

from which we obtain

$$
\frac{d y}{d x}=-\frac{A x+B y}{B x+C y} \text {. }
$$

Thus $d y / d x=0$ when $x=-B y / A$. Substituting this expression for $x$ into (2.1) we find

$$
y= \pm \frac{1}{\sqrt{C-B^{2} / A}} \text { and hence } \quad W=\frac{2}{\sqrt{C-B^{2} / A}}
$$

Finally we can write down the ratio

$$
r=\frac{W}{w}=\frac{2}{w \sqrt{C-B^{2} / A}}=\frac{1}{b \sqrt{C-B^{2} / A}}
$$


TABLE 1. Comparison of computed and experimental values of $r$ and $\theta ; a / b=1.36$, lateral $\mathrm{x}$-rays.

\begin{tabular}{|c|c|c|c|c|c|}
\hline rotation & $r$ & \multicolumn{2}{|c|}{$r$ (from x-rays) } & \multicolumn{2}{c|}{$\theta$ (computed) } \\
$\theta_{\mathrm{e}}$ & (computed) & supination & pronation & supination & pronation \\
\hline 10 & 1.013 & 1.014 & 1.017 & 10.3 & 11.7 \\
20 & 1.049 & 1.037 & 1.074 & 17.3 & 25.2 \\
30 & 1.101 & 1.107 & 1.134 & 31.0 & 35.5 \\
40 & 1.162 & 1.148 & 1.173 & 37.8 & 41.7 \\
50 & 1.224 & 1.228 & 1.218 & 50.6 & 48.9 \\
60 & 1.280 & 1.298 & 1.275 & 64.0 & 59.1 \\
70 & 1.323 & 1.343 & 1.320 & 76.7 & 69.2 \\
80 & 1.351 & 1.356 & 1.356 & 83.8 & 83.8 \\
90 & 1.360 & 1.360 & 1.360 & 90.0 & 90.0 \\
\hline
\end{tabular}

Substituting for $A, B$ and $C$ in (2.2) we find, after some manipulation,

$$
r=\sqrt{\frac{a^{2}}{b^{2}}+\left(1-\frac{a^{2}}{b^{2}}\right) \cos ^{2} \theta} .
$$

For the angle of rotation $-\pi / 2 \leq \theta \leq \pi / 2$ given $r, a$ and $b$, we rearrange (2.3) to give

$$
\theta= \pm \arccos \sqrt{\frac{a^{2} / b^{2}-r^{2}}{a^{2} / b^{2}-1}}
$$

choosing the sign of $\theta$ in accordance with the external evidence.

If it is preferable to determine $\ell$ and $L$ from an $x$-ray and so obtain the ratio $\rho=L / \ell$ instead of $r=W / w$, then we can simply interchange $a$ and $b$ in (2.3) to give an equation for $\rho$ and, similarly, we may interchange $a$ and $b$ and substitute $\rho$ for $r$ in (2.4) to obtain an expression for the angle of rotation $\theta$.

\section{Comparison with experiment}

$\mathrm{X}$-rays of a fractured forearm are taken with the arm in either full supination or full pronation, in which positions it is assumed that the major axis of the radius above the break is horizontal. Then in each of these two positions an x-ray may be taken from two different directions to give two different views: the lateral view which shows the ratio $r=w / W$, or the antero-posterior (AP) view which shows the ratio $\rho=\ell / L$. Experimental data has been obtained for each of these four possible combinations of arm position/x-ray view for a fractured radius of aspect ratio $a / b=1.36$ [1]. 
TABLE 2. Comparison of computed and experimental values of $\rho$ and $\theta ; a / b=1.36$, AP x-rays.

\begin{tabular}{|c|c|c|c|c|c|}
\hline rotation & $\rho$ & \multicolumn{2}{|c|}{$\rho$ (from X-rays) } & \multicolumn{2}{c|}{$\theta$ (computed) } \\
$\theta_{\mathrm{e}}$ & (computed) & supination & pronation & supination & pronation \\
\hline 10 & 0.993 & 0.990 & 0.995 & 12.3 & 8.8 \\
20 & 0.973 & 0.970 & 0.978 & 21.0 & 17.8 \\
30 & 0.941 & 0.961 & 0.950 & 24.1 & 27.5 \\
40 & 0.900 & 0.899 & 0.902 & 40.3 & 39.5 \\
50 & 0.855 & 0.859 & 0.839 & 49.1 & 53.4 \\
60 & 0.810 & 0.836 & 0.788 & 54.0 & 65.3 \\
70 & 0.771 & 0.793 & 0.764 & 64.1 & 72.1 \\
80 & 0.745 & 0.742 & 0.740 & 81.5 & 83.1 \\
90 & 0.735 & 0.735 & 0.735 & 90.0 & 90.0 \\
\hline
\end{tabular}

TABLE 3. Computed rotation angle $\theta_{\mathrm{av}}$ as the average of angles determined from lateral and AP $\mathrm{x}$-rays with arm in supination, pronation and both.

\begin{tabular}{|c|c|c|c|}
\hline $\begin{array}{c}\text { rotation } \\
\theta_{\mathrm{e}}\end{array}$ & supination & pronation & both \\
\hline 10 & 11.3 & 10.3 & 10.8 \\
20 & 19.1 & 21.5 & 20.3 \\
30 & 27.5 & 31.5 & 29.5 \\
40 & 39.0 & 40.6 & 39.8 \\
50 & 49.8 & 51.2 & 50.5 \\
60 & 59.0 & 62.2 & 60.6 \\
70 & 70.4 & 70.6 & 70.5 \\
80 & 82.6 & 83.4 & 83.0 \\
90 & 90.0 & 90.0 & 90.0 \\
\hline
\end{tabular}

Table 1 gives the correspondence between the angle of rotation and the ratio $r=W / w$ for lateral $\mathrm{x}$-rays with the arm in supination and pronation. For a given experimental rotation $\theta_{\mathbf{e}}$ (Column 1) the ratio $r$ computed by (2.3) is given in Column 2 . Columns 3 and 4 give $r$ as determined from x-rays taken with the arm in supination and pronation. These values show good correspondence with the computed value of $r$. The values of $r$ determined from $\mathrm{x}$-rays may then be used in (2.4) to compute the rotation angle $\theta$ as given in Columns 5 and 6 - which, in practice, is the derived information of primary importance. An indication of the usefulness of the elliptic approximation is obtained by comparing these angles against $\theta_{\mathrm{e}}$ in Column 1 . The comparison is best shown graphically as in Figure 4 where the relation (2.4) between $r$ and $\theta$ is shown as a solid curve and the experimental $(r, \theta)$ data are plotted as points. Table 2 gives similar information to that in Table 1 but for AP $\mathrm{x}$-rays while Figure 5 


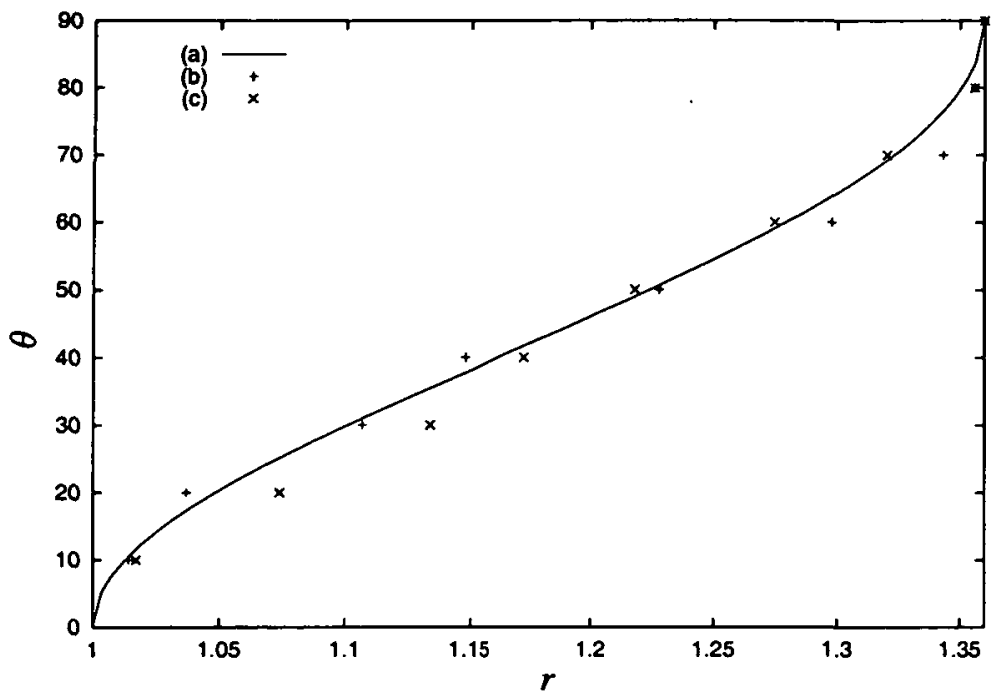

FIGURE 4. Ratio $r$ versus rotation $\theta ; a / b=1.36$, lateral $\mathrm{x}$-rays (refer Table 1). (a) $\theta=$ $\arccos \sqrt{\left(a^{2} / b^{2}-r^{2}\right) /\left(a^{2} / b^{2}-1\right)}$, (b) experimental data, supination, (c) experimental data, pronation.

graphically shows the comparison between theory and experiment.

While there is a need for comparison with much more data before drawing any definitive conclusions as to how the equations yielded by an elliptic approximation to the radius cross-section might be used by an orthopaedic surgeon, they are certainly in good agreement with the data presented. With a sufficiently good estimate of the aspect ratio of the bone $(a / b)$, a single $x$-ray might be sufficient for an initial assessment of the degree of rotation. If this should indicate a rotation well under or well above the critical point of functional impairment the desirability of corrective surgery would be easily established.

For borderline cases (say with a rotation of around $30 \pm 5^{\circ}$ ) further $x$-rays giving different views of the break could be taken and computed rotation angles averaged as shown in Table 3. Column 2 of this table gives, for each $\theta_{e}$ and for the arm in supination, the arithmetic mean of the rotation angles determined from lateral and AP $x$-rays (Column 5 of Tables 1 and 2). Column 3 gives the same average, but for the arm in pronation (Column 6 of Tables 1 and 2). Column 4 gives $\theta$ as the arithmetic mean of all four computed angles determined from lateral and AP x-rays with the arm in supination and pronation. The angle so determined compares exceedingly well with the experimental rotation $\theta_{\mathrm{e}}$, as seen in Figure 6. Only for $\theta_{\mathrm{e}}=80^{\circ}$ do we obtain an average of the four computed rotation angles that is uncharacteristic in having an inaccuracy of $3^{\circ}$ while all other results are accurate to within $1^{\circ}$. This may indicate some error in the experimental setup and measurement rather than inaccuracy due to 


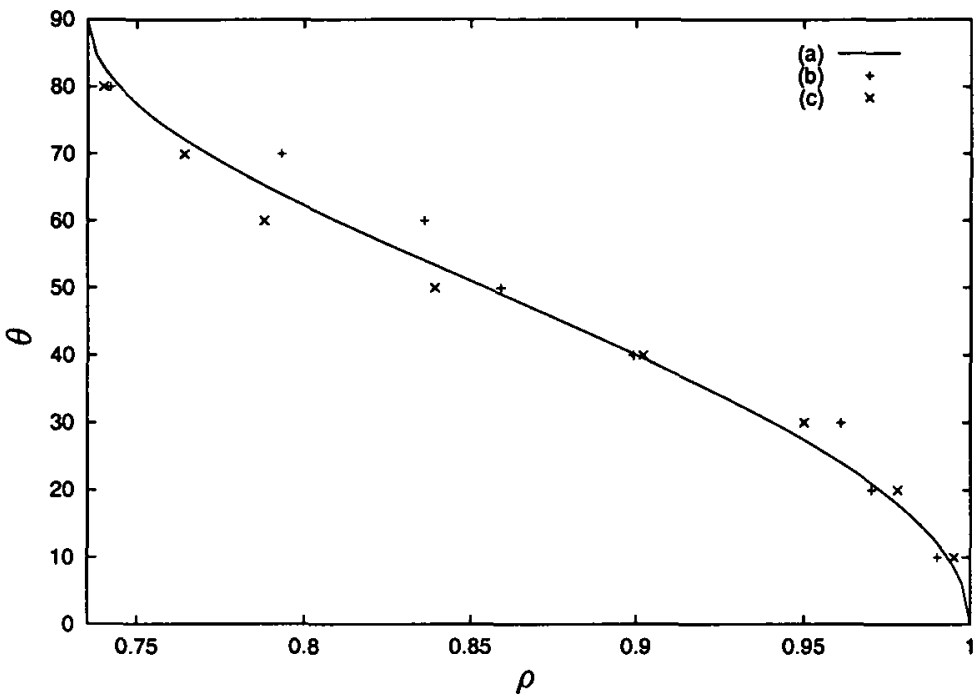

FIGURE 5. Ratio $\rho$ versus rotation $\theta ; a / b=1.36$, AP $x$-rays (refer Table 2). (a) $\theta=$ $\arccos \sqrt{\left(b^{2} / a^{2}-\rho^{2}\right) /\left(b^{2} / a^{2}-1\right)}$, (b) experimental data, supination, (c) experimental data, pronation.

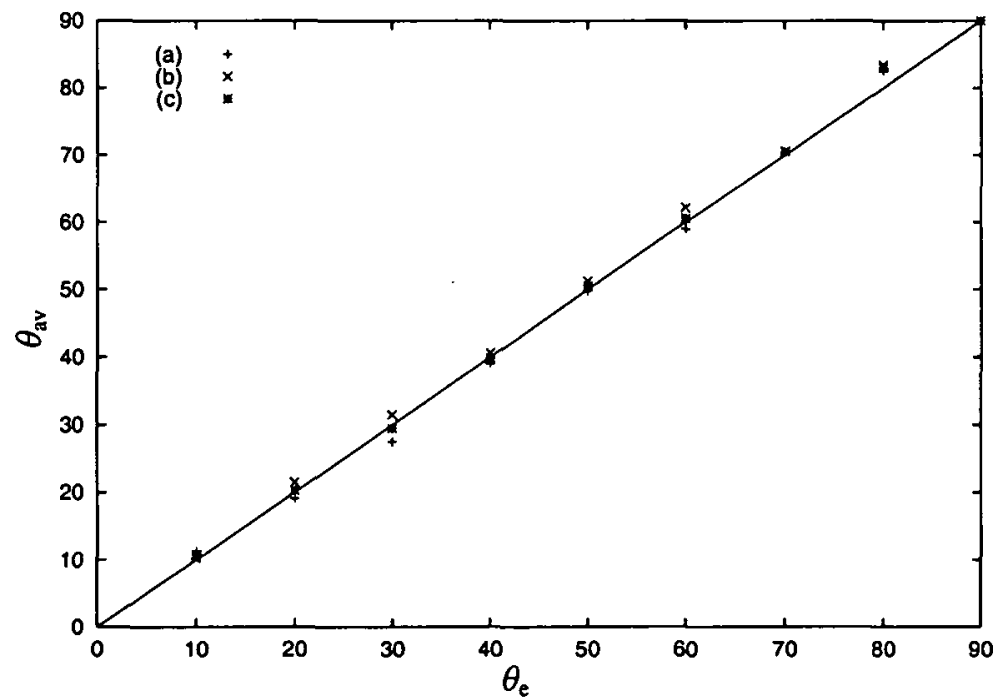

FIGURE 6. Average computed rotation $\theta_{\mathrm{av}}$ versus experimental rotation $\theta_{\mathrm{c}}$ : (a) supination, (b) pronation and (c) both (refer Table 3). 
non-ellipticity of the bone cross-section. In any case, any rotation above $50^{\circ}$ must be in the range of essential corrective surgery.

\section{Conclusion}

Formulae relating rotation angle and the ratio of widths seen in a two-dimensional side view of rotated and unrotated ellipses have been obtained and applied to the problem of determining rotation of the radius in a forearm fracture. Initial comparisons of rotations computed using these formulae with experimental data are sufficiently good as to encourage a much more extensive investigation which is to be conducted by others [1]. It is expected that the formulae will prove very useful to orthopaedic surgeons.

\section{Acknowledgement}

I am indebted to Dr Ed Bateman for first bringing this problem to my attention and then providing substantial experimental data.

\section{References}

[1] E. Bateman, Orthopaedic Department, Royal North Shore Hospital, Sydney. Private communication.

[2] J. E. Frazer, Anatomy of the human skeleton, 6th ed. (J. \& A. Churchill Ltd, London, 1965). 